\title{
The generalized and extended uncertainty principles and their implications on the Jeans mass
}

\author{
${ }^{1}$ H. Moradpour*, ${ }^{1}$ A. H. Ziaie ${ }^{\dagger},{ }^{1}$ S. Ghaffari ${ }^{\ddagger}$ and ${ }^{2}$ F. Feleppa $\S$ \\ ${ }^{1}$ Research Institute for Astronomy and Astrophysics of Maragha (RIAAM), P.O. Box 55134-441, Maragha, Iran and \\ 2 Department of Physics, University of Trieste, via Valerio 2, 34127 Trieste, Italy
}

\begin{abstract}
The generalized and extended uncertainty principles affect the Newtonian gravity and also the geometry of the thermodynamic phase space. Under the influence of the latter, the energy-temperature relation of ideal gas may change. Moreover, it seems that the Newtonian gravity is modified in the framework of the Rényi entropy formalism motivated by both the long-range nature of gravity, and the extended uncertainty principle. Here, the consequences of employing the generalized and extended uncertainty principles, instead of the Heisenberg uncertainty principle, on the Jeans mass are studied. The results of working in the Rényi entropy formalism are also addressed. It is shown that unlike the extended uncertainty principle and the Rényi entropy formalism which lead to the same increase in the Jeans mass, the generalized uncertainty principle can decrease it. The latter means that a cloud with mass smaller than the standard Jeans mass, obtained in the framework of the Newtonian gravity, may also undergo the gravitational collapse process.
\end{abstract}

\section{INTRODUCTION}

The Heisenberg uncertainty principle (HUP) is in full agreement with the Bekenstein entropy (BE) which can produce the Newtonian gravity (NG) (Ali \& Tawfik 2013; Ali 2014; Awada \& Ali 2014a; Awada \& Ali 2014b; Majumder 2011; Srednicki 1993; Verlinde 2011; Wang et al. 2009). NG has a crucial role in getting some well-known criteria appeared in study the structure formation problems such as those of Chandrasekhar and Jeans (Longair 1998). In this regard, it has been shown that the generalized and extended uncertainty principles, namely GUP and EUP, respectively, modify NG and can also affect the Chandrasekhar limit (Ong 2018; Ong \& Yao 2018). Indeed, GUP and EUP change the Bekenstein limit of entropy (Ali \& Tawfik 2013; Ali 2014; Awada \& Ali 2014a; Awada \& Ali 2014b; Majumder 2011; Moradpour et al. 2019; Wang et al. 2009), and even, EUP gives a motivation to use the Rényi entropy formalism instead of the Boltzmann-Gibbs (BG) entropy to study the gravitational systems (Moradpour et al. 2019).

The quantum gravity scenarios generally propose (Bolan \& Cavaglia 2005; Bambi \& Urban 2008; Park 2008; Kempf et al. 1995; Nozari \& Fazlpour 2006)

$$
\Delta x \Delta p \geq \frac{\hbar}{2}\left[1+\beta(\Delta x)^{2}+\eta(\Delta p)^{2}+\gamma\right],
$$

where $\beta, \eta$ and $\gamma$ are positive and $\gamma$ can depend on the expectation values of $p$ and $x$. Smallest uncertainties are obtained for $\gamma=0$ (Nozari \& Fazlpour 2006), and nonzero minimal uncertainty in both position and momentum is guaranteed whenever $\beta$ and $\eta$ are positive (Kempf et al. 1995; Nozari \& Fazlpour 2006). Throughout this

\footnotetext{
*h.moradpour@riaam.ac.ir

†ah.ziaie@riaam.ac.ir

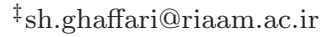

§feleppa.fabiano@gmail.com
}

paper, we set $\gamma=0$ (Nozari \& Fazlpour 2006), and although some authors call Eq. (1) as GUP (Kempf et al. 1995; Nozari \& Fazlpour 2006), by following Refs. (Ali \& Tawfik 2013; Ali 2014; Awada \& Ali 2014a; Awada \& Ali 2014b; Majumder 2011; Moradpour et al. 2019; Wang et al. 2009) and (Bolan \& Cavaglia 2005; Bambi \& Urban 2008; Park 2008), we call the $\beta=0$ and $\eta=0$ cases as GUP and EUP, respectively. It has been shown that the accelerated universe and the galaxies flat rotation curves may be modelled within the frameworks of GUP and EUP without considering any odd energy source, namely dark energy and dark matter (Awada \& Ali 2014b; Moradpour et al 2017; Moradpour et al 2018a; Moradpour et al. 2019b; Komatsu 2017), a result also signalling us to a deep connection between the fine and large structures. Finally, it is worthwhile mentioning that $\beta$ may also reveal the non-extensive features of gravitational systems (Moradpour et al. 2019), and Eq. (1) affects the thermodynamics of early universe (Nozari \& Fazlpour 2006).

The gravitational potential energy of a cloud with mass $M$ and radius $R$, formed due to gravitational potential $V(r)$, is evaluated as

$$
U=\int_{0}^{M} V(r) d M
$$

In the framework of NG, where $V_{N}(r)=-G \frac{M}{r}$, the gravitational potential energy $\left(U_{N}\right)$ is obtained as

$$
U_{N}=-\int_{0}^{R} \frac{G M}{r} 4 \pi \rho(r) r^{2} d r=-\frac{3 G M^{2}}{5 R},
$$

written by assuming that the cloud density is constant (or equally $\rho(r)=\rho_{0} \equiv$ const). In the framework of BG entropy, as a special case of Shannon entropy, the kinetic energy $(K)$ of a cloud, approximated by an ideal gas composed of $N$ non-interacting particles with mass $\mu$ (and thus $M=N \mu$ ) at temperature $T$, is 


$$
\langle K\rangle=\frac{3}{2} N K_{B} T,
$$

where $K_{B}$ denotes the Boltzmann constant. Based on the Virial theorem, the cloud is in Virial equilibrium if $\langle K\rangle=-\frac{1}{2} U$, and gravitational collapse may happen if $\langle K\rangle<-\frac{1}{2} U$ leading to

$$
N K_{B} T<\frac{G M^{2}}{5 R},
$$

for NG. Using the $R=\left(\frac{3 M}{4 \pi \rho_{0}}\right)^{\frac{1}{3}}$ relation in rewriting Eq. (5), one reaches

$$
M>M^{J} \equiv\left(\frac{5 K_{B} T}{G \mu}\right)^{\frac{3}{2}}\left(\frac{3}{4 \pi \rho_{0}}\right)^{\frac{1}{2}},
$$

as the lower bound of the cloud mass to collapse. Any change in the Jeans mass affects the fragmentation of cloud (Forgan \& Rice 2011), and in fact, the predictions about the structure formation at different scales (Capozziello et al 2012; Roshan \& Abbassi 2014; Vainio \& Vilja 2016; de Martino \& Capolupo 2017). As an example, while the mass of CB 188, as a Bok globule, is smaller than $M^{J}$, it has a protostar (Kandori et al 2005; Vainio \& Vilja 2016) meaning that new physics, such as modified gravity, is unavoidable to decrease the Jeans mass (Vainio \& Vilja 2016).

As we saw, NG and the ordinary extensive statistical mechanics, based on Shannon entropy, form the backbone of the obtained $M^{J}$, and on the other, Newtonian force is the direct result of using BE allowed by HUP (Ali \& Tawfik 2013; Ali 2014; Awada \& Ali 2014a; Awada \& Ali 2014b; Majumder 2011; Moradpour et al 2019; Srednicki 1993; Verlinde 2011; Wang et al. 2009). In fact, NG is the weak field limit of general relativity, and the modified versions of general relativity, such as the $f(R)$ (Capozziello et al 2012), scalar-tensor-vector (Roshan \& Abbassi 2014) and Eddington-inspired Born-Infield gravities (de Martino \& Capolupo 2017), may decrease the Jeans mass by increasing the Newtonian force at the weak field limit. Additionally, it also seems that the consequences of non-zero values of $\beta$ and $\eta$ are not limited to the fine structure, and indeed, their effects can even be seen at the cosmic and galaxies scales (Awada \& Ali 2014b; Komatsu 2017; Moradpour et al 2017; Moradpour et al 2018a; Moradpour et al 2019; Moradpour et al. 2019b; Nozari \& Fazlpour 2006), and the physics of black hole (Moradpour et al 2019; Ong 2018; Ong \& Yao 2018), white dwarf and neutron stars (Ong 2018; Ong \& Yao 2018).

Moreover, it has been claimed that the generalized entropies, such as the Rényi entropy, should be used instead of the Shannon entropy to study the systems including long-range interactions such as gravity (Rényi 1970; Masi 2005; Moradpour et al 2017; Moradpour et al 2018a; Moradpour et al. 2019b; Komatsu 2017). In fact, even the ideal gas can give us permission to use the generalized entropies such as those of the Rényi and Tsallis instead of the Shannon entropy (Biró 2013). The Jeans mass changes whenever instead of the BG entropy, Tsallis entropy is considered to determine the thermodynamic quantities of system (Jiulin 2004; Lima et al 2002). As we previously mentioned, it also seems that a deep connection between the Rényi statistics and the fine structure parameters such as $\beta$, appeared in EUP, may exist (Moradpour et al. 2019). The above arguments motivate us to study the Jeans mass in the Rényi entropy formalism.

In the next section, we firstly study the effects of EUP on $M^{J}$. Additionally, motivated by the fact that there is a deep connection between EUP and Rényi statistics (Moradpour et al. 2019), the implications of the Rényi entropy on the Jeans mass are also addressed in Sec. (II). The consequences of GUP on $M^{J}$ are studied in third section, where it is also shown that GUP may help us in explaining the existence of star in some Bok globules. The last section is devoted to a summary. We also follow the $c=\hbar=G=K_{B}=1$ units.

\section{EUP AND JEANS INSTABILITY: THE ROLE OF RÉNYI ENTROPY}

Whenever $\eta=0$, EUP is obtained from Eq. (11) as (Bolan \& Cavaglia 2005; Bambi \& Urban 2008; Park 2008; Kempf et al. 1995; Ong \& Yao 2018; Wang et al. 2009)

$$
\Delta x \Delta p \geq \frac{1}{2}\left[1+\beta(\Delta x)^{2}\right],
$$

which modifies the BE entropy $\left(\frac{A}{4}\right)$ as (Moradpour et al 2019)

$$
S=\frac{\pi}{4 \beta} \ln \left(1+\beta \frac{A}{\pi}\right)
$$

where $A=4 \pi r^{2}$ denotes the system boundary located at radius $r$. Clearly, $\mathrm{BE}$ is recovered at the $\beta=0$ limit (Moradpour et al 2019). It is also useful to mention that, mathematically, the above entropy is called the Rényi entropy (Moradpour et al 2019).

Using the entropic force approach, one can reach (Moradpour et al 2018b)

$$
F_{S}=-\frac{M m}{r^{2}} \frac{1}{4 \beta r^{2}+1}
$$

instead of NG $\left(F_{N}=-\frac{M m}{r^{2}}\right)$. In agreement with the asymptotic behavior of $S$, it is also obvious that NG is recovered for $\beta=0$. Bearing the $F=-m \frac{d V}{d r}$ relation in mind, the modified Newtonian potential $V_{s}$ is obtained as 


$$
\begin{aligned}
& V_{S}(r)=-\frac{1}{m} \int F_{s} d r \\
& =-M\left[\frac{1}{r}+2 \sqrt{\beta}\left(\tan ^{-1}(2 \sqrt{\beta} r)-\frac{\pi}{2}\right)\right],
\end{aligned}
$$

where the integration constant has been chosen to meet the $V_{S}(r \rightarrow \infty)=0$ condition. Now, applying this potential to a cloud with mass $M$ and density $\rho_{0}$, and by following the recipe led to Eq. (3), we get

$$
\begin{aligned}
& U_{S}=\int_{0}^{R} V_{S}(r) d M=-\frac{3 M^{2}}{5 R}+2 \sqrt{\beta} U_{c}=U_{N}+2 \sqrt{\beta} U_{c}, \\
& U_{c}=-\int_{0}^{R} M\left(\tan ^{-1}(2 \sqrt{\beta} r)-\frac{\pi}{2}\right) d M=U_{N} R D, \\
& D=\frac{5}{6}\left(\frac{\left(1+\xi^{6}\right) \tan ^{-1}(\xi)}{\xi^{6}}+\frac{5 \xi^{2}-3 \xi^{4}-15}{15 \xi^{5}}-\frac{\pi}{2}\right), \\
& \xi=2 \sqrt{\beta} R,
\end{aligned}
$$

for the corresponding gravitational potential energy which can briefly be written as $U_{S}=U_{N}(1+\xi D)$.

The Helmholtz free energy $(A)$ of a monatomic ideal gas which consists of $N$ particles with mass $\mu$ at temperature $T$ and satisfies EUP (7) is obtained as (Chung \& Hassanabadi 2019)

$$
A=-T\left[N \ln \left(4 \pi(2 \mu \pi T)^{\frac{3}{2}} V_{e f f}\right)-\ln (N !)\right]
$$

where

$$
V_{\text {eff }}=\frac{4 \pi}{\beta}\left[\left(\frac{3 V}{4 \pi}\right)^{\frac{1}{3}}-\frac{1}{\beta^{\frac{1}{2}}} \tan ^{-1}\left(\sqrt{\beta}\left(\frac{3 V}{4 \pi}\right)^{\frac{1}{3}}\right)\right],
$$

in which $V$ denotes the container volume (Chung \& Hassanabadi 2019). Using $P=-\left(\frac{\partial A}{\partial V}\right)_{T, N}$, one can see the ideal gas law $(P V=N T)$ is modified in this situation (Chung \& Hassanabadi 2019). Now, following the $S_{g}=-\left(\frac{\partial A}{\partial T}\right)_{V, N}$ and $K=A+T S_{g}$ relations, one reaches

$$
K=\frac{3}{2} N T,
$$

for the internal energy of ideal gas nothing but Eq. (4). Here, $S_{g}$ represents also the gas entropy which differs from that of the system boundary (8) (Ali \& Moussa 2014; Moradpour et al 2019; Moradpour et al. 2014).

As an example to verify the consequences of $\beta$, consider the $4 \beta=\frac{1}{R^{2}}$ case which leads to $U_{S} \approx \frac{5}{18} U_{N}$, and thus the corresponding Jeans mass

$$
M_{S}^{J}(\xi=1)=\left(\frac{18 T}{\mu}\right)^{\frac{3}{2}}\left(\frac{3}{4 \pi \rho_{0}}\right)^{\frac{1}{2}}
$$
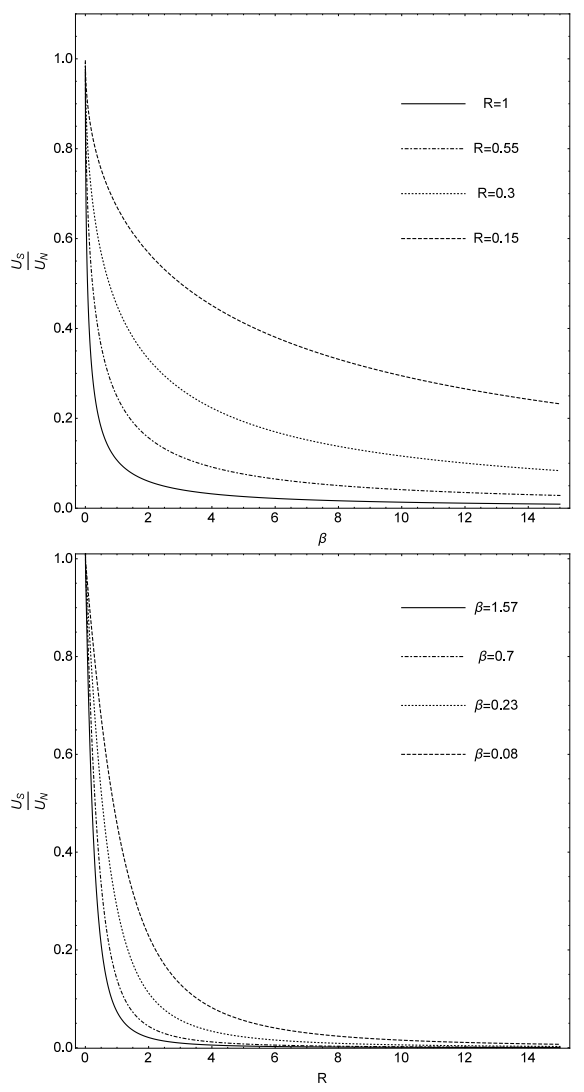

FIG. 1: Upper panel: plot of the ratio $\frac{U_{S}}{U_{N}}$ as a function of $\beta$ parameter for different values of $R$. Lower panel: $\frac{U_{S}}{U_{N}}$ versus $R$ for some values of $\beta$.

which is always bigger than $M^{J}$. It is worthwhile to mention here that the Jeans mass may also be increased in the framework of the Eddington-inspired Born-Infield gravity (De Martino \& Capolupo 2017). In fact, at a constant radius $R$, the Jeans mass is increased with increasing $\beta$. Mathematically, as it is obvious from Fig. 1, it is due to the fact that the $\xi D$ term is negative and decreases with increasing $\delta(0<|\xi D|<1)$ meaning that $U_{S}$ is always bigger than $U_{N}$ for $\beta>0$. In this manner, since both $U_{S}$ and $U_{N}$ are negative, we have $0<\frac{U_{S}}{U_{N}}<1$.

In general, if we have $2 \sqrt{\beta}=\frac{\zeta}{R}$, where $\zeta$ is constant, then $\xi=\zeta=$ constant, and we get the following expression for the Jeans mass

$$
M_{S}^{J}=\left(\frac{5 T}{\mu(1+\zeta D)}\right)^{\frac{3}{2}}\left(\frac{3}{4 \pi \rho_{0}}\right)^{\frac{1}{2}}>M^{J} .
$$

Now, consider a system consisting of $W$ discrete states while the $i^{\text {th }}$ state can be occupied with probability $P_{i}$. It has also been proposed that systems including long-range interactions, such as gravity, may follow the power-law probability distribution $P_{i}^{q}$ instead of the ordinary distribution $P_{i}$, where $q$ is an unknown parameter evaluated by using experiments and probably the other parts of 
physics (Rényi 1970; Masi 2005). Indeed, even an ideal gas can provide the power-law probability distribution $P_{i}^{q}$ (Biró 2013). In this regard, the Rényi entropy corresponding to the probability distribution $P_{i}^{q}$ is defined as (Rényi 1970; Masi 2005)

$$
\mathcal{S}=\frac{1}{\delta} \ln \sum_{i=1}^{W} P_{i}^{1-\delta}
$$

where $\delta \equiv 1-q$, and the Shannon entropy is recovered at the $\delta \rightarrow 0$ limit (Masi 2005). This entropy finally leads to (Moradpour et al 2018b)

$$
\mathcal{S}=\frac{1}{\delta} \ln \left(1+\delta \frac{A}{4}\right)
$$

and

$$
\mathcal{F}=-\frac{M m}{r^{2}} \frac{1}{\delta \pi r^{2}+1}
$$

for the horizon entropy and the corresponding modified NG, respectively (Komatsu 2017; Moradpour et al 2017; Moradpour et al 2018b; Moradpour et al 2019). From mathematical point of view, Eqs. (18) and (19) are obtainable replacing $4 \beta$ in Eqs. (8) and (9) with $\delta \pi$, respectively. One can easily check that this recipe is also followable to find the modified Newtonian potential and the corresponding gravitational potential energy from Eqs. (10) and (11), respectively.

Although entropy, probability distribution and the phase space geometries used to obtain Eqs. (4) and (14) (Chung \& Hassanabadi 2019) differ from those based on Eq. (17) and the power-law probability distribution $P_{i}^{q}$ (Parvan \& Biró 2005), it has been shown that the ideal gas still satisfies Eq. (14) in the Rényi formalism (Parvan \& Biró 2005). Thus, the results obtained by employing EUP are also valid here, and one can get the exact from of the outcomes by replacing $4 \beta$ with $\delta \pi$.

\section{GUP AND JEANS INSTABILITY}

For $\beta=0$, Eq. (1) leads to (Kempf et al. 1995)

$$
\Delta x \Delta p \geq \frac{1}{2}\left[1+\eta(\Delta p)^{2}\right]
$$

which modifies the Newtonian potential corresponding to mass $M$ as (Awada \& Ali 2014b)

$$
V_{G U P}(r)=-\frac{M}{r}\left[1+\frac{\Delta}{r^{2}}\left(\frac{2}{3}+\ln \left(4 \pi r^{2}\right)\right)\right],
$$

where $\Delta=\frac{\eta}{144}$. Now, using Eq. (2), one can get
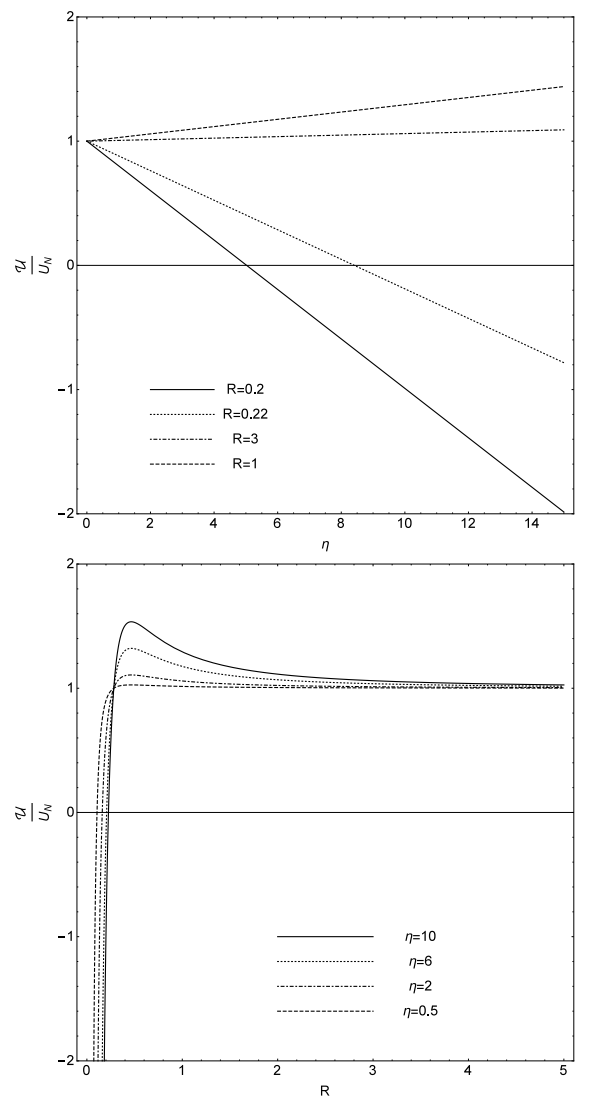

FIG. 2: Upper panel: plot of the ratio $\frac{\mathcal{U}}{U_{N}}$ as a function of $\eta$ parameter for different values of $R$. Lower panel: $\frac{\mathcal{U}}{U_{N}}$ versus $R$ for some values of $\eta$. Note that whenever $\mathcal{U}<U_{N}$, since both $\mathcal{U}$ and $U_{N}$ are negative, we have $\frac{\mathcal{U}}{U_{N}}>1$.

$$
\begin{aligned}
& \mathcal{U}=\int_{0}^{R} V_{G U P}(r) d M=U_{N}+\Delta \mathcal{U}_{c} \\
& \mathcal{U}_{c}=-\int_{0}^{R} \frac{M}{r^{3}}\left(\frac{2}{3}+\ln \left(4 \pi r^{2}\right)\right) d M=U_{N} B \\
& B=\frac{5}{3 R^{2}} \ln \left(4 \pi R^{2}\right),
\end{aligned}
$$

for the gravitational potential energy $(\mathcal{U})$ corresponding to a cloud with mass $M$, density $\rho_{0}$ and radius $R$. It has been shown that, up to the first order in $\eta$, GUP modifies Eq. (4) as (Motlaq \& Pedram 2014)

$$
K=\frac{3}{2} N T-3 \eta N \mu T^{2},
$$

where $\mu$ denotes again the mass of the ideal gas particles. More studies on the thermodynamic properties of this gas, such as its pressure, can be found in (Motlaq \& Pedram 2014; Miraboutalebi \& Matin 2014). Bearing the Virial theorem in mind, collapse will happen if the cloud mass $M$ satisfies 
$\frac{10 T\left(\frac{1}{2 \mu}-\eta T\right)}{\left(\frac{4 \pi \rho_{0}}{3}\right)^{\frac{1}{3}}} \leq M^{\frac{2}{3}}\left[1+\frac{5 \eta}{342\left(\frac{3 M}{4 \pi \rho_{0}}\right)^{\frac{2}{3}}} \ln \left(4 \pi\left(\frac{3 M}{4 \pi \rho_{0}}\right)^{\frac{2}{3}}\right)\right](.24)$

Clearly, Eq. (6) is recovered at the appropriate limit $\eta=$ 0. Moreover, since $\eta$ is finite (Kempf et al. 1995; Nozari \& Fazlpour 2006; Nozari \& Etemadi 2012) and $M \gg 1$, the above equation takes approximately the form

$$
\frac{10 T\left(\frac{1}{2 \mu}-\eta T\right)}{\left(\frac{4 \pi \rho_{0}}{3}\right)^{\frac{1}{3}}} \leq M^{\frac{2}{3}}
$$

In this situation the Jeans mass $\left(\mathcal{M}^{J}\right)$ is obtained as

$$
\mathcal{M}^{J} \approx M^{J}(1-2 \eta \mu T)^{\frac{3}{2}}
$$

meaning that the Jeans mass is decreased and is positive only when $\eta \mu T<\frac{1}{2}$. In fact, depending on the value of $\eta \mu T$, the ratio $\frac{\mathcal{M}^{J}}{M^{J}}$ can be sensible.

While some Bok globules, as the interstellar clouds of dust and gas, include star or at least experience the star formation process (Bourke et al 1995; Kandori et al 2005; Launhardt et al 2000; Launhardt et al 2013; Vainio \& Vilja 2016; Yun and Clemens 1990), their mass is less than their corresponding Jeans mass (Kandori et al 2005; Vainio \& Vilja 2016). This fact motivates physicists to use modified gravity in order to revise Jeans mass for describing the observations (Vainio \& Vilja 2016).

As an example, consider the Bok globule CB 188 with mass $M_{C B} 188\left(\simeq 7 \cdot 19 \mathrm{M}\right.$.) and $M^{J} \simeq 7 \cdot 7 \mathrm{M}$., where $\mathrm{M}$. denotes the Sun mass, which is at temperature $T=19$ in Kelvin scale, and includes a protostar (Vainio \& Vilja 2016). In this situation, assuming $M_{C B} 188=\mathcal{M}^{J}$, it is easy to see $\frac{\ln \left(\mathcal{M}^{J}\right)}{\left(\mathcal{M}^{J}\right)^{\frac{2}{3}}}$ is negligible and thus Eq. (26) is available which leads to

$$
\eta_{C B 188} \simeq \frac{0.0012}{\mu}
$$

as a primary estimation for the value of $\eta$ in CB 188 . In fact, an upper bound on $\mathcal{M}^{J}$ corresponds to a lower bound on $\eta$, and for values bigger than $\eta_{C B} 188$, we have $\mathcal{M}^{J}<M_{C B}$ 188. For a number of Bok globules, including stars (Kandori et al 2005), following the above recipe, the minimum values of $\eta$ have been addressed in Table. 1. Here, we assumed that the host cloud is composed of $N$ identical particle with mass $\mu$. In reality, interstellar clouds include a range of different particles, and therefore, the mass of the particle with most abundance in the host cloud may be considered as a primary estimation for $\mu$. In the case of CB 188, since it includes a protostar (Vainio \& Vilja 2016), we follow Vainio \& Vilja (2016) and approximate $\mu$ with the Hydrogen mass $\left(\sim 10^{9} \mathrm{eV} \sim 10^{-19} \mathrm{M}_{\mathrm{P}}\right)$, where $\mathrm{M}_{\mathrm{P}}$ denotes the
TABLE I: Approximation values for the minimum values of $\eta$ in some of the Bok globules by using the information of Vainio \& Vilja (2016). Here, $M$ and $T$ denote the mass and temperature of Bok globules, respectively.

\begin{tabular}{ccccc} 
Bok globule & $T$ & $\frac{M}{M_{*}}$ & $\frac{M^{J}}{M_{*}}$ & $\eta \simeq \frac{1-\left(\frac{M}{M J}\right)^{\frac{2}{3}}}{2 \mu T}$ \\
\hline CB 87 & 11.4 & $2.73 \pm 0.24$ & 9.6 & $\frac{0.0249}{\mu}$ \\
\hline CB 110 & 21.8 & $7.21 \pm 1.64$ & 8.5 & $\frac{0.0024}{\mu}$ \\
\hline CB 131 & 25.1 & $7.83 \pm 2.35$ & 8.1 & $\frac{0.0004}{\mu}$ \\
\hline CB 161 & 12.5 & $2.79 \pm 0.72$ & 5.4 & $\frac{0.1424}{\mu}$ \\
\hline CB 184 & 15.5 & $4.70 \pm 1.76$ & 11.4 & $\frac{0.0144}{\mu}$ \\
\hline FeSt $1-457$ & 10.9 & $1.12 \pm 0.23$ & 1.4 & $\frac{0.0063}{\mu}$ \\
\hline Lynds 495 & 12.6 & $2.95 \pm 0.77$ & 6.6 & $\frac{0.0165}{\mu}$ \\
\hline Lynds 498 & 11.0 & $1.42 \pm 0.16$ & 5.7 & $\frac{0.0275}{\mu}$
\end{tabular}

Plank mass $\left(\mathrm{M}_{\mathrm{P}} \equiv \sqrt{\frac{\hbar c}{G}}=1\right.$ in our units, and we have $\mathrm{M}_{\mathrm{P}} \sim 10^{-8} \mathrm{Kg}$ in the SI units), which finally leads to $\eta_{C B} 188 \sim 10^{16}$. If we apply this assumption to all cases studied in Table. 1, then the maximum value of $\eta$ is obtained for CB 161 as $\mu \sim 10^{18}$ meaning that for values of $\eta$ bigger than $10^{18}, \mathcal{M}^{J}$ of those Bok globules studied in this paper is smaller than their mass.

It is also worthwhile to mention that, in the presence of the equivalence principle and in the SI units, we have $\eta<2.3 \times 10^{60}$ (Feng et al 2017). Following Feng et al (2017), simple calculations lead to $\eta<6.8 \times 10^{61}$ in our units. The minimum upper bound on $\eta$ has also been reported as $\eta<10^{21}$ (Das \& Vagenas 2008; Feng et al 2017). Finally, it should be noted that a simple recipe to include the effect of the mass of all ingredients of Bok globule is to model the Bok globule as a system with $N$ particles of mass $\bar{\mu}$ such that $i) N \equiv \sum_{i} N_{i}$, and $i i$ ) $\bar{\mu} \equiv \frac{\sum_{i} N_{i} \mu_{i}}{N}$, where $N_{i}$ and $\mu_{i}$ denote the number and mass of the $i^{\text {th }}$ type particle, respectively. It means that, since Hydrogen is the lightest atom, we have $\bar{\mu}>\mu$ if there is something other than Hydrogen in the cloud, and therefore, the obtained minimum values of $\eta$ will be decreased by considering all ingredients of the Bok globules.

In fact, since $\eta$ is positive (Kempf et al. 1995; Motlaq \& Pedram 2014; Nozari \& Fazlpour 2006), we have $\Delta B>0$ if $R^{2}>\frac{1}{4 \pi}$ which leads to $\mathcal{U}<U_{N}$ meaning that the Jeans mass is decreased in this situation (see Fig. 2). Moreover and in agreement with Fig. 2, although the deviation of $\frac{\mathcal{U}}{U_{N}}$ from 1 is sensible for $R<3$, depending 
on the values of temperature, $\eta$ and $\mu$, the $\frac{\mathcal{M}^{J}}{M^{J}}$ quantity may be appreciable. It is due to this fact that while $\frac{\mathcal{U}}{U_{N}}$ is independent of temperature, Jeans mass depends on it.

\section{SUMMARY}

The implications of GUP and EUP on the Jeans mass have been studied. The results indicate that, compared with the Newtonian regime, the gravitational potential energy of the cloud is decreased when EUP is valid, leading to growth in the Jeans mass. It has been argued that since gravity is a long-range interaction, one may use the Rényi entropy formalism to study the gravitational systems (Komatsu 2017; Moradpour et al 2017; Moradpour et al 2018a; Moradpour et al 2018b; Moradpour et al. 2019b). This entropy (18) is also confirmed by EUP (Moradpour et al 2019), and leads to Eq. (19) (Moradpour et al 2018b). Additionally, as we addressed, Eq. (14) is valid in both the EUP and Rényi frameworks. Hence, the EUP results, obtained in this paper, are also available in the framework of Rényi entropy (one just needs to replace $4 \beta$ in the results of second section with $\delta \pi$ to obtain the corresponding results in the Rényi formalism).
Moreover, we found out that GUP can increase the gravitational potential energy which reduces the Jeans mass. Therefore, GUP suggests that gravitational collapse can happen for clouds with mass smaller than the ordinary Jeans mass obtained from NG $\left(M^{J}\right)$. The Bok globules observations confirm the existence of collapse process in clouds whose their mass is smaller than $M^{J}$ (Kandori et al 2005; Launhardt et al 2000; Vainio \& Vilja 2016). Fitting such observations to the modified Jeans mass obtained from GUP, one may find some primary estimations for $\eta$ (For example Eq. (27)). The evolution of fragments and perturbations (in both astrophysical and cosmic scales) under the shadow of the GUP modifications to Jeans mass are also another interesting subjects, which are out of the scope of this project, and are followable in the subsequent works.

\section{Acknowledgment}

We are grateful to the anonymous reviewer for worthy comments. The work of H. Moradpour has been supported financially by Research Institute for Astronomy \& Astrophysics of Maragha (RIAAM) under research project No. 1/6025 -55 .
[1] Ali A. F., Moussa M., 2014, AHEP 2014, 629148

[2] Ali A. F., Tawfik A., 2013, AHEP 2013, 126528

[3] Ali A. F., 2014, Phys. Lett. B 732, 335

[4] Awada A., Ali A. F., 2014a, JHEP 06, 093

[5] Awada A., Ali A. F., 2014b, Cent. Eur. J. Phys. 12(4), 245

[6] Bambi C., Urban F. R., 2008, Class. Quantum. Grav. 25, 095006

[7] Biró T. S., 2013, Physica A 392, 3132

[8] Bolen B., Cavaglia M., 2005, Gen. Relativ. Gravit. 37, 1255

[9] Bourke T. L., Hyland A. R., Robinson G., 1995, MNRAS 276, 1052

[10] Capozziello, S., et al., 2012, Phys. Rev. D 85, 044022

[11] Chung W. S., Hassanabadi H., 2019, Int. J. Mod. Phys. A 34, 1950041

[12] Das S., Vagenas E. C., 2008, Phys. Rev. Lett. 101, 221301

[13] De Martino, I., Capolupo, A., 2017, Eur. Phys. J. C 77, 715

[14] Feng Z. W., et al., 2017, Phys. Lett. B 768, 81

[15] Forgan D., Rice K., 2011, Mon. Not. R. Astron. Soc. 417, 1928

[16] Jiulin D., 2004, Physica A 335, 107

[17] Kandori R., et al., 2005, Astron. J. 130, 2166

[18] Kempf A., Mangano G., Mann R. B., 1995, Phys. Rev. D 52, 1108

[19] Komatsu N., 2017, Eur. Phys. J. C 77, 229

[20] Launhardt R., et al., 2000, In Birth and Evolution of Binary Stars, IAU Symp. No. 200, ed. B. Reipurth, H. Zinnecker, pp. 1035. San Francisco: Astron. Soc. Pac.

[21] Launhardt R., et al., 2013, A \& A 551, A 98

[22] Lima J. A. S., Silva R., Santos J., 2002, Astron. Astro- phys. 396, 309

[23] Longair M. S., 1998, Galaxy Formation, Springer Berlin Heidelberg

[24] Majumder B., 2011, Astrophys. Space. Sci. 336, 331

[25] Masi M., 2005, Phys. Lett. A 338, 217

[26] Miraboutalebi S., Farhang Matin L., 2014, Can. J. Phys. $93(5), 574$

[27] Moradpour H., et al., 2014, AHEP 2014, 718583

[28] Moradpour H., et al., 2017, Phys. Rev. D 96, 123504

[29] Moradpour H., et al., 2018a, Eur. Phys. J. C 78, 829

[30] Moradpour H., et al., 2018b, Phys. Lett. B 783, 82

[31] Moradpour H., et al., 2019, arXiv:1902.01703

[32] Moradpour H., et al., 2019b, arXiv:1902.10202

[33] Motlaq M. A., Pedram P., 2014, J. Stat. Mech. P08002

[34] Norazi K., Etemadi A., 2012, Phys. Rev. D 85, 104029

[35] Norazi K., Fazlpour B., 2006, Gen. Relativ. Gravit. 38, 1661

[36] Ong Y. C., 2018, JCAP 09, 015

[37] Ong Y. C., Yao Y., 2018, Phys. Rev. D 98, 126018

[38] Park M. I., 2008, Phys. Lett. B 659, 698

[39] Parvan A. S., Biró T. S., 2005, Phys. Lett. A 340, 375

[40] Rényi A., 1970, Probability Theory, North-Holland, Amsterdam

[41] Roshan M. Abbassi, S., 2014, Phys. Rev. D, 90, 044010

[42] Srednicki M., 1993, Phys. Rev. Lett. 71, 666

[43] Vainio J., Vilja I., 2016, Gen. Relativ. Gravit. 48, 129

[44] Verlinde E. P., 2011, JHEP 04, 029

[45] Wang F. J., Gui Y. X., Zhang Y., 2009, Gen. Relativ. Gravit. 41, 2381

[46] Yun J. L., Clemens D. P., 1990, Astrophys. J. Lett. 365, L73 